\title{
A Hybrid Approach for QoS enabled Web Service Retrieval
}

\author{
P. Gayathri, K. Priyanka and I.R. Praveen Joe
}

\begin{abstract}
Earlier, web services are used to retrieve data for small amount of user only, but now a day's more data retrieved through web services by more number of users in all the actions of day to day life. There are numerous web services available in the internet to collect the data. Today internet plays a vital role in every body's life. Internet provides all the information one can access through the web service. To make the retrieval more efficient, Hidden Markov Models (HMM) \&Mediator Agent Model are used in the project. The Hidden Markov Models can be used to measure and predict the behavior of Web Services in terms of response time, and thus can be used to rank services quantitatively. The mediator agent Model is used to select web services with the help of ranking method. The Mediator Agent Model will provide web service to the user from the web service provider. It will be processed in such a way that optimal result is obtained. If many users refer the optimal site then server loading will occur to reduce that problem server redirection is used in this project.
\end{abstract}

Index Terms--- Hidden States, Probability Prediction, UDDI, Web Service Selection

\section{INTRODUCTION}

$\mathrm{T}$ ODAY'S dynamic environment there is various companies from all around the world compete over different offerings to the user. They provide more adversaries for the organizations growth through the Web Service. There are some aspects hidden from the user, they cannot able to predict the behavior of the web service in terms of QoS factors. User can only select the web service after they visited, they can't able to predict the best web service because of some hidden aspect (eg: Bad node, Database, calling another web service).There is no standard way for the user to predict the best among the equal functional web services. This paper provides a standard way to measure and predict the WS behavior in terms of response time. Service Oriented architecture systems Reliability heavily based on various underling technologies for web services, computing environment and unpredictable environment.

P. Gayathri, Department of Computer Science and Engineering, KCG College of Technology, Chennai, Tamil Nadu, India. E-mail:gayathribanu26@gmail.com

K. Priyanka, Department of Computer Science and Engineering, KCG College of Technology, Chennai, Tamil Nadu, India. E-mail:kspriyankacse@gmail.com

I.R. Praveen Joe, Assistant Professor, Department of Computer Science and Engineering, KCG College of Technology, Chennai, Tamil Nadu, India. E-mail:praveen.joe.it@kcgcollege.com DOI: 10.9756/BIJIEMS.8141
This can be predicted by using the following:

1. A directed graph is built among hidden states of composition web services are used.

2. Current status of each vertex of directed graph is analyzed i.e., underlying hidden states.

3. The time interval for each web service behavior is predicted for hidden states' in terms of response time.

4. The selection of optimal web services used in composition based on hidden states behavior.

\section{RELATED WORK}

Today's dynamic and unpredictable environment, it is difficult to analyze the QoS Parameters in WS. There are more researchers proposed various methods and frameworks for web service Selection. Here we have summarized some of the methods for the related work.

Waseem Ahamed [1] have produced a novel approach for web service selection based on response time in which the prediction of web service is done with the help of HMM. The Viterbi algorithm is used to predict the optimal path and Baum-Welch algorithm is used to train the probability estimation.

Divyasachan [2] have produced a model called mediator agent model which suggest the best WS from the web service registry by using Ranking method which is done with the help of page weighting method.

N.HemaPriya [3] has produced a fuzzy rule based algorithm for web service selection and web service composition. Which is used to select the appropriate service with composite of QoS Parameters.

Moussa Kaouan [4] released a paper "Shared Repository based approach for storing and discovering web service. Which uses UDDI for web service discovery and storage. They proposed a matching algorithm for performing the task between equal functional WS.

\section{Proposed System}

In the proposed method the best web service is selected through the probability prediction of each state. Once the probability prediction is done with HMM. The optimal path is generated for each state in the web service. Through this path the redirection of server is done. The ranking of each web service is done in a way that feedback from each user is stored. Because the optimality of the web service is done only through the feedback of the user in this model. The mediator agent model is used to retrieve the web service which is stored with the best response time. Then it provides the link to the user for the best web service. The user can search the web 
service by entering the keyword user and the visitor will be provided with the services according to the priority of the sites that available in web. Hence the user-friendly services are achieved.

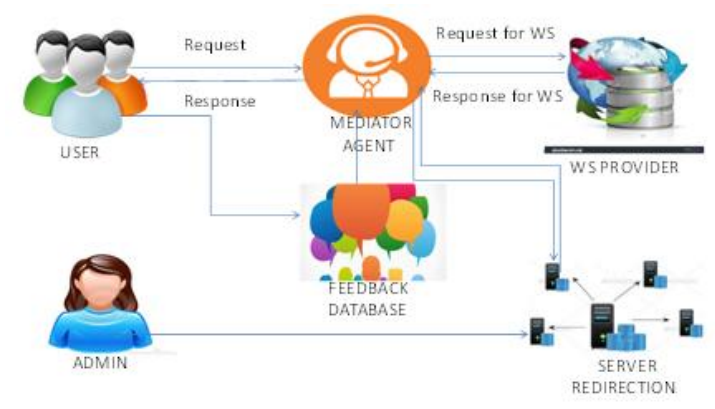

Figure 3.1: System Architecture

\section{EXPERIMENT}

Various available web services are taking into account for generating the graph which shows the optimal result for the composite web service

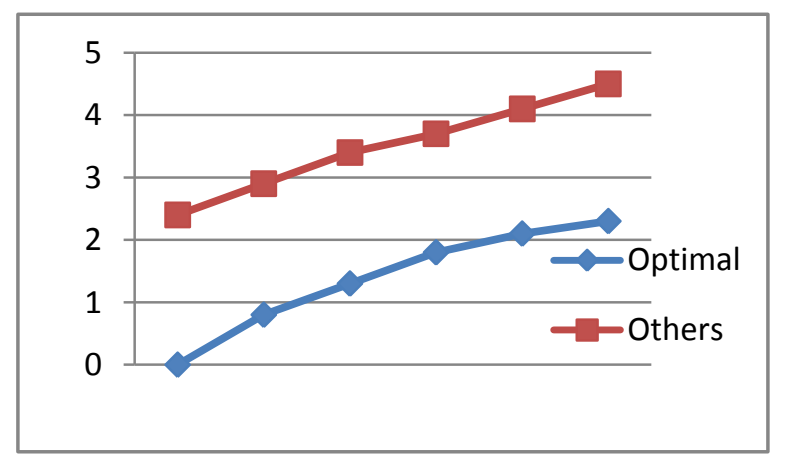

Figure 4.1: Response Time Graph

\begin{tabular}{|l|l|l|}
\hline \multicolumn{2}{|c|}{ Time Taken for each Service } & Available Web Services \\
\hline Optimal & Others & \\
\hline 0 & 2.4 & Web service 1 \\
\hline 0.8 & 2.9 & Web service 2 \\
\hline 1.3 & 3.4 & Web service 3 \\
\hline 1.8 & 3.7 & Web service 4 \\
\hline 2.1 & 4.1 & Web service 5 \\
\hline 2.3 & 4.5 & Web Service 6 \\
\hline
\end{tabular}

Figure 4.2: Response Time Result

For each web service there is a change in response time with response time. But the threshold value of optimal service is optimal than others. Response Time of each Web Service is calculated below,

Response Time rt $=$ Latency + Execution

Time of each WS.

\section{RESULT}

A set of functionally equivalent web services are selected and run automatically in the proposed system. The QoS values are selected based upon the response time. The graph which is shown in the above will easily calculate the best web service. The best services are shown, when the user enter the keyword to search for the web service.

\section{FUTURE WORK}

In The future Work we have the idea to take more QoS Parameter in this paper (e.g: Security, availability, Throughput, Transmission delay).We would like to continue our research in the field of web service selection .Further QoS based parameter along with HMM can be collaborated with Security to produce more efficiency .

\section{CONCLUSION}

In this paper a method called HMM is used to predict the behavior of the web service. The user can easily get the best web service with the help of this method. Here server redirection is also available to avoid the time delay. The mediator agent will rank every web service in terms of response time. The user can get the best web service from this method.

\section{REFERENCES}

[1] Waseem Ahmed, Yongwei Wu, Member, IEEE, WeiminZheng. "Response Time Based Optimal Web Service Selection"

[2] DivyaSachan, Saurabh Kumar Dixit, Sandeep Kumaran, "Qos aware formalized model for Semantic web service selection" International Journal of Web and Semantic Technology, Indian Institute of Technology Roorkee, India.

[3] Muhammad Fahad, NejibMoalla and Yacine Ourzout, "Dynamic Execution of a Business Process Via Web Service Selection and Orchestration",

[4] Passent El-Kafrawy, EmadElabd and HanaaFathi, "A Trustworthy Reputation Approch for Web Service Discovery"

[5] Mehrnoosh Kheradmand, HomayunMotameni, "An Approch to web Service Selection Based on Composite QoS Parameters"

[6] R.Sethuraman, Dr T.Sasiprabha, A.Sandhya, "An Effective QoS Based web Service Composition Algorithm for Integration of Travel \& Tourism Resources".

[7] Tongguang Zhang, "QoS-aware Web Service based on Particle Swarm Optimization".

[8] Valentin Kilmov, "Peculiarities of Semantic Web Services Cloud Runtime".

[9] N.HemaPriya, A.M.ShobanaPriya and S.Chandramathi, "QoS based Selection and Composition of Web Services-A Fuzzy Approch".

[10] MoussaKaouan, DjelloulBouchiha and Sidi Mohamed Bensilimane, "Shared repository Approach for Sharing and Discovering Web Service". 